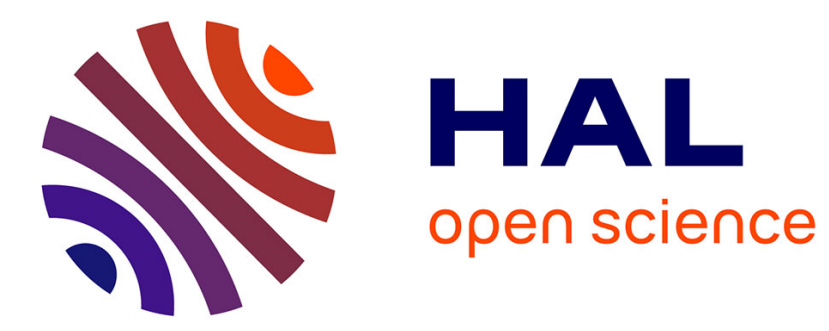

\title{
Motion control architecture of a 4-fin U-CAT AUV using DOF prioritization
}

\author{
Taavi Salumäe, Ahmed Chemori, Maarja Kruusmaa
}

\section{To cite this version:}

Taavi Salumäe, Ahmed Chemori, Maarja Kruusmaa. Motion control architecture of a 4-fin U-CAT AUV using DOF prioritization. IROS: Intelligent RObots and Systems, Oct 2016, Daejeon, South Korea. pp.1321-1327, 10.1109/IROS.2016.7759218 . lirmm-01723924

\section{HAL Id: lirmm-01723924 https://hal-lirmm.ccsd.cnrs.fr/lirmm-01723924}

Submitted on 5 Mar 2018

HAL is a multi-disciplinary open access archive for the deposit and dissemination of scientific research documents, whether they are published or not. The documents may come from teaching and research institutions in France or abroad, or from public or private research centers.
L'archive ouverte pluridisciplinaire HAL, est destinée au dépôt et à la diffusion de documents scientifiques de niveau recherche, publiés ou non, émanant des établissements d'enseignement et de recherche français ou étrangers, des laboratoires publics ou privés. 


\title{
Motion control architecture of a 4-fin U-CAT AUV using DOF prioritization
}

\author{
Taavi Salumäe ${ }^{1}$, Ahmed Chemori ${ }^{2}$, and Maarja Kruusmaa ${ }^{1}$
}

\begin{abstract}
This paper demonstrates a novel motion control approach for biomimetic underwater vehicles with pitching fins. Even though these vehicles are highly maneuverable, the actuation of their different degrees of freedom (DOFs) is strongly coupled. To address this problem, we propose to use smooth DOF prioritization depending on which maneuver the vehicle is about to do. DOF prioritization has allowed us to develop a modular, easily applicable and extendable motion control architecture for U-CAT vehicle, which is meant for archaeological shipwreck penetration. We demonstrate the benefits of this architecture by developing an remotely operated vehicle autopilot for depth and yaw using a nonlinear state feedback controller. We also show the extensibility of the approach by controlling 3 DOFs of a fully autonomous U-CAT. The real-time experimental results show high position tracking precision (depth RMS error: 1.9 cm; yaw RMS error: $2.5^{\circ}$ ) Comparative experiments justify the use of DOF prioritization.
\end{abstract}

\section{INTRODUCTION}

Many marine researchers, such as underwater archaeologists and biologists, employ unmanned underwater vehicles in their everyday work. These vehicles mostly have time withstood and proven propeller-based designs. These designs are justified in most traditional underwater vehicle tasks, but their applicability is limited to relatively simple conditions (i.e. open waters). They are usually not usable in shallow waters, confined areas, in proximity of divers or animals etc. To develop underwater vehicles for these more complex situations, several researchers have taken inspiration from different animal species. For example there exist fish robots for mariculture monitoring [1], snake robots for amphibious missions [2], and even an aerial-aquatic diving bird robot [3]. One class of biomimetic underwater vehicles consists of robots that use multiple actuated fins in a configuration that gives them high maneuverability. Most of these vehicles can be classified as turtle-like robots. Turtle-like robots use 4 fins with 1 to 3 rotational degrees of freedom (DOFs). Some of the earliest examples are "Turtle 2005" [4] of Kogakuin University and the robot turtle of Nanyang Technological University [5]. Both of them use rolling and pitching fore fins for propulsion and pitching hind fins for control. "Finnegan the RoboTurtle" [6] developed in MIT/WHOI uses 4 rolling and pitching fins instead of 2 giving the robot a hovering capability and improving maneuverability. To even more increase the similarity to turtle, the "Naro-tartaruga" robot [7] adds additional DOFs of yaw to its fore fins. "Gen" series

\footnotetext{
${ }^{1}$ Authors are with Centre for Biorobotics, Tallinn University of Technology, Tallinn, Estonia, taavi.salumaedttu.ee

${ }^{2}$ Author is with LIRMM UMR CNRS/Univ. of Montpellier, 161 rue Ada, 34392 Montpellier, France, ahmed. chemori@lirmm. fr
}

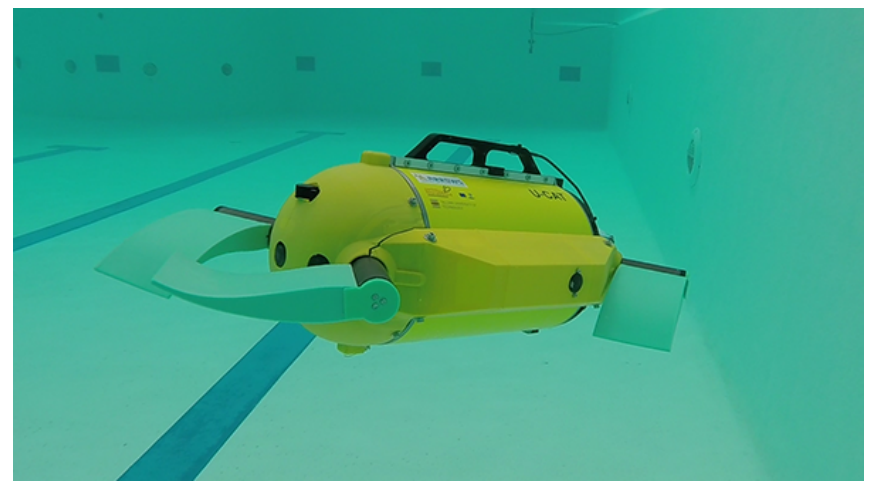

Fig. 1. U-CAT biomimetic 4-fin AUV.

vehicles [8] of Naval Research Laboratory use different, so called active shape deformation fins.

All these turtle inspired vehicles have better maneuverability than most traditional autonomous underwater vehicles (AUVs). However, they generate thrust mostly using the roll motion of fins, giving the vehicles much better performance in surge than in other degrees of freedom. Therefore, this kind of locomotion is not optimal for missions where the vehicle has to perform equally along the different degrees of freedom. For example wall-following, close-up inspection and investigation of confined spaces. Another major drawback of turtle-like locomotion is the complexity of multipleDOF fin actuators. Such actuators are large and less reliable.

These problems are partly solved with another class of multiple-fin underwater vehicles. It consists of vehicles that do not try to mimick exact turtle propulsion, even though some authors still call them turtle-like robots. They are usually actuated by single-DOF pitching fins. Probably the most known example is the "Madeline" of Nekton research [10], which was used to show that it is more efficient to swim using only fore fins [11]. The "Madeline's" commercialised successor is the IRobot "Transphibian" [12] designed for amphibian remotely operated vehicle (ROV) missions. Very similar design to "Transphibian" has been used on another robot developed in the Robotics Institute of Beihang University [13]. A different amphibious robot design of Peking university [14] uses fins that are actuated by two parallel motors through special five-bar link mechanism. The mechanism allows to pitch the fins for swimming, but also to use them for walking on ground. Peking University has developed another four-fin robot with pitching and yawing fins [15] which make the vehicle fully actuated. While all the previous vehicles use 4 fins, the Aqua vehicle of McGill 
University has 6 pitching fins [16]. The Aqua's fins can be used either for crawling or swimming.

As the fin locomotion is different from propeller locomotion, all the above mentioned robots also need different methods for motion control. The main difference between control of multiple pitching fin robots and traditional highly maneuverable underwater vehicles is that the latter ones mostly use separate actuator for controlling every degree of freedom. In case of multi-fin vehicles, different degrees of freedom cannot be actuated directly, but have to be controlled through different combinations of fin motions. This creates strong coupling between the control of different DOFs. For example, when a vehicle is diving at full speed, its fins are pointing up. Therefore, the robot cannot surge at the same time. Most of the fin-actuated vehicles do not deal with this issue. Many of them present only open-loop or manual control [4], [5], [13]-[15]. Popular approach is to use central pattern generators to achieve the desired fin motion [13][15]. Other researchers have concentrated on the control of the vehicles where different DOFs are coupled to dominant surge motion [6], [7]. In some cases they have achieved good results. For example [6] demonstrates that using unstable roll and pitch is extremely beneficial for turning during forward motion. Other articles describe the automatic position control only for $1 \mathrm{DOF}$ at the same time [8], [15]. In [10] 3 DOFs are simultaneously controlled. The authors do not report any coupling issues, but our test with similar configuration may suggest their presence. The most advanced control has been developed for the Aqua vehicles [17]-[22]. On Aqua a combination of model-free PD and PI controllers is used to control yaw, roll, pitch and heave. A gain scheduling technique is used to modify the gains of these controllers depending on the surge speed [20]. Even though authors have reported good trajectory following, this was achieved by tuning an array of 45 control parameters ( 9 parameters at 5 different speeds) [20]. They have tried to simplify the process by using online gain adaptation algorithms [21], however the presented results show relatively large deviations from desired attitude.

In this paper we demonstrate a more easily applicable motion control approach for 4-fin biomimetic vehicles. We present a modular, easily extensible control architecture, which can be applied to fully autonomous control, but also to ROV autopilot. We propose a method for dealing with DOF coupling problem by prioritizing the control of different DOFs. The method assigns continuous priorities to different DOF controllers depending on what the vehicle is about to do. This is achieved by reducing the priority of action of some controllers if an action of a more prioritized controller increases.

We demonstrate the applicability of the proposed control architecture on a small-size U-CAT vehicle developed for operating in confined spaces. We use the architecture for implementing depth and yaw autopilot including modelbased state feedback linearization control, but we also show the usage of the system for fully autonomous 3 DOF motion. Real-time experiments show precise trajectory tracking and large improvement with respect to cases without DOF prioritization.

\section{U-CAT PLATFORM}

U-CAT is a biomimetic underwater vehicle designed for autonomous and semi-autonomous inspection of confined areas, such as shipwrecks, caves and man-made underwater structures. It was developed in the framework of European Commission funded research project ARROWS [23]. The aim of the ARROWS project was to adapt and develop low-cost AUV technologies to significantly reduce the cost of archaeological operations. U-CAT was designed specifically keeping in mind the archaeologists requirements for unmanned shipwreck penetration missions. It uses fin-based locomotion to achieve high maneuverability and more quiet and safe locomotion. More detailed description of the vehicle and the justification of fin-based design is given in [24]. One unique feature of U-CAT compared to other mentioned 4-fin vehicles lies in its fin placement. The fins are placed in a way that allows to create thrust forces in all the 6 degrees of freedom. This includes sway, which cannot be controlled with other vehicles that use 1 DOF pitching fins, but which is extremely useful for various video inspection tasks. Some preliminary control results for 1 degree of freedom using non model-based controllers are published in [25].

U-CAT is $56 \mathrm{~cm}$ long and weights approximately $19 \mathrm{~kg}$. Its four fins are actuated by $60 \mathrm{~W}$ Maxon brushless DC motors driven by Maxon Epos motor drivers using sensorless backEMF feedback control. The vehicle has an internal battery allowing at least 6 hours of autonomous operation. The whole control architecture is developed on an internal single-board computer with ARM Cortex A9 quad-core $1 \mathrm{Ghz}$ processor. We use modular development approach with the help of ROS middleware. The on-board computer communicates with a remote PC through a tether. We use the remote PC only for forwarding remote controller actions in ROV mode, and for logging into the vehicle through $\mathrm{SSH}$ for debugging, development and data collection. As a remote controller we used Sony Playstation DualShock 3.

In this paper we use the following sensors of U-CAT:

- Invensense MPU-6050 IMU for measuring attitude. In the current study only yaw measurement is directly used as a feedback in control loop. The roll and pitch measurements are used only for coordinate transformations. The sampling frequency in our setup is $5 \mathrm{~Hz}$. Even though the frequency is relatively low, experimental result show it is enough to achieve stable control. This is probably due to low reaction rate of the fin's thrust.

- GEMS 3101 analog output pressure sensor with 18-bit DAQ for measuring depth. The sampling frequency is $10 \mathrm{~Hz}$ and the resolution is $0.06 \mathrm{~cm}$.

U-CAT has some additional devices which we did not use in this study, but which will be used in further developments: Applicon acoustic modem for underwater communication and localization; two active buoyancy control modules; custom-developed hydrophone array for acoustic beacon localization; custom-made echosounder array for 
close-distance obstacle avoidance; Point Grey Chameleon camera; and various system health monitoring sensors.

\section{A. Dynamics}

We model the rigid body dynamics of the vehicle in 6 DOFs using Fossen's robot-like vectorial model of marine craft [26].

$$
\begin{gathered}
\dot{\eta}=J(\eta) \nu \\
M \dot{\nu}+C(\nu) \nu+D(\nu) \nu+g(\eta)=\tau
\end{gathered}
$$

where $\boldsymbol{\eta}=[x, y, z, \varphi, \vartheta, \psi]^{T}$ is the vector of positions in the earth-fixed frame, $\boldsymbol{\nu}=[u, v, w, p, q, r]^{T}$ is the vector of velocities in the body-fixed frame and $\boldsymbol{J}(\boldsymbol{\eta}) \in \mathbb{R}^{6 \times 6}$ is the transformation matrix mapping from the body-fixed frame to the earth-fixed frame. $M$ is the system inertia matrix including added mass, $\boldsymbol{C}(\boldsymbol{\nu})$ is the Coriolis-centripetal matrix, $\boldsymbol{D}(\boldsymbol{\nu})$ is the damping matrix, $\boldsymbol{g}(\boldsymbol{\eta})$ is the vector of gravitational/buoyancy forces and moments and $\tau$ is the vector of control inputs. The vector $\tau$ is defined as $\boldsymbol{\tau}=\boldsymbol{B F}$, where $\boldsymbol{F} \in \mathbb{R}^{4}$ is the vector of thrust forces generated by the fins and $\boldsymbol{B}$ describes the fin configuration. The thrust of each fin $F_{i}$ is a complex relationship depending on the softbody dynamics coupled to hydrodynamics. For simplification purposes we model the thrust $F_{i}$ with an empirical equation

$$
F_{i}=\xi\left(, A_{i}, f_{i}, \Phi_{i}\right)
$$

where the fin oscillation amplitude $A_{i}$ and oscillation frequency $f_{i}$ define the average thrust magnitude and the offset angle $\Phi_{i}$ defines the thrust direction. We identified the average magnitude of (2) experimentally by mounting the fin module on a waterproof 4-axis force sensor and actuating with different amplitudes and frequencies. Continuous surface fit describes the average thrust in the direction of $\Phi_{i}$ depending on the actuation frequency and amplitude.

Initial model parameters $M, C(\nu), D(\nu)$ and $g(\eta)$ came from theoretical calculations using a precise CAD model. The model includes density of components allowing to calculate inertia. Strip theory allows finding the added mass and approximated drag describes damping. To achieve higher modeling precision in the most important DOFs, we experimentally identified surge, sway, heave and yaw components of $\boldsymbol{M}$ and $\boldsymbol{D}(\boldsymbol{\nu})$. We actuated the vehicle with known constant forces in different directions and measured its reactions using internal sensors and external overhead camera.

The centre of buoyancy of U-CAT is above the centre of mass making the robot relatively stable in pitch and roll. For this paper the vehicle is trimmed to be neutrally buoyant.

\section{PROposed CONTROL ARCHITECTURE}

The general structure of the proposed control architecture is shown in Fig. 2. In this article we concentrate on the simultaneous control of 3 DOFs, however the control is presented and implemented in general matrix form. Matrix form implementation allows fast extensibility if control of additional DOFs is required. As U-CAT is mechanically

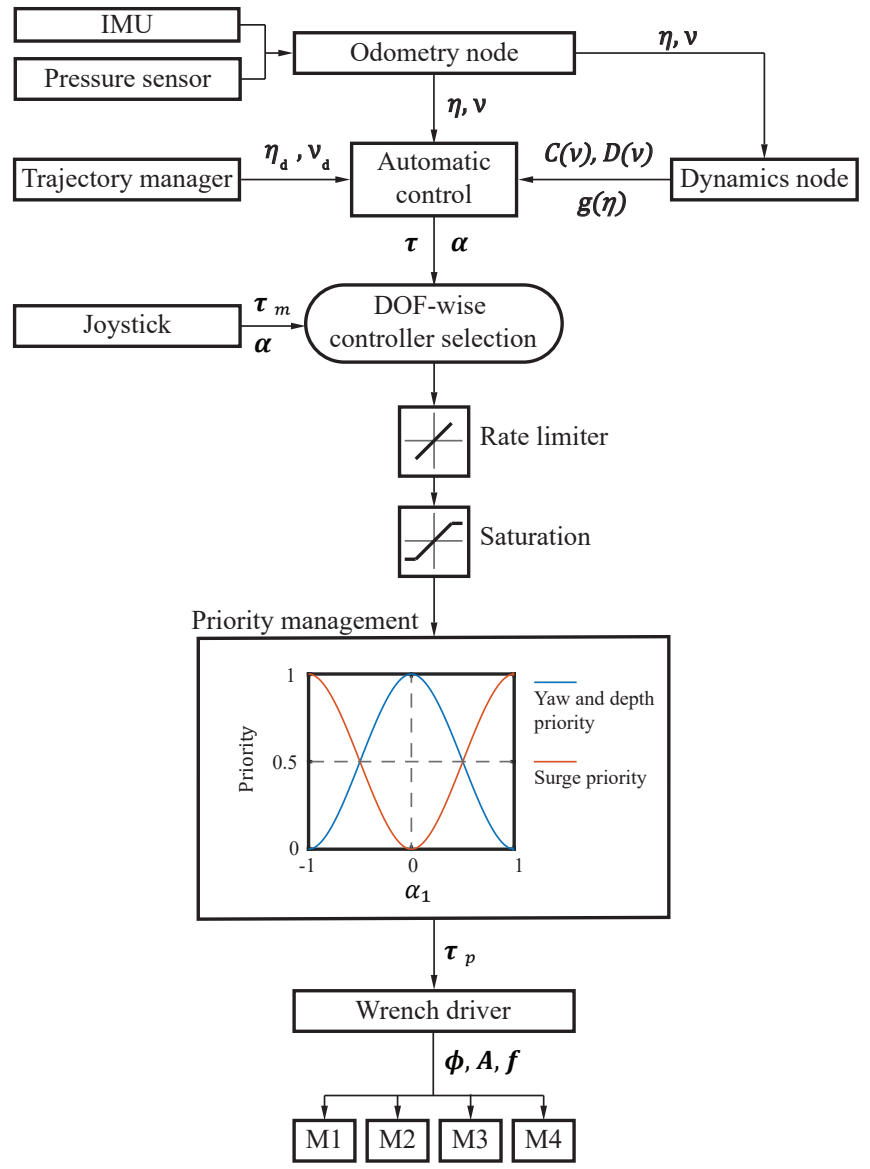

Fig. 2. Motion control architecture using DOF prioritization

relatively stable in roll and pitch we did not concentrate on the control of these DOFs at this stage.

The main idea behind the proposed control is that we develop separate controllers for different DOFs. We then prioritize the control actions with smooth functions to reduce the actuation coupling problem. The controllers can be individually tuned and replaced with different control method or with manual inputs from remote controller. Our system is implemented as a set of independent nodes: Automatic control for different DOFs, Dynamics node, Priority management, Wrench driver, Motor control, Odometry node, Trajectory manager and Human intervention.

\section{A. Automatic control}

To choose the most suitable control approach we have tested different model-free and model-based controllers on U-CAT. For this article we chose to use an inverse dynamics compensation with acceleration feedforward [27]. This controller showed the best performance in a nominal case where robot's dynamical parameters were constant during experiment. The idea behind the inverse dynamics compensation is based on cancelling nonlinear terms of the vehicle dynamics, allowing the linear control action (i.e. PID) to only work on minimizing tracking error. The method is widely used in robot manipulators, but also in underwater robotics and even on some biomimetic underwater vehicles [28]. 
More specifically we implemented a state feedback linearization described in [26]. The control law is defined as

$$
\tau=M a^{b}+n(\nu, \eta)
$$

where $\boldsymbol{n}$ is the nonlinear compensation vector

$$
n(\nu, \eta)=C(\nu) \nu+D(\nu) \nu+g(\eta)
$$

and $\boldsymbol{a}^{\boldsymbol{b}}$ is the commanded acceleration in body-fixed frame. The commanded acceleration vector is chosen as PIDcontroller with acceleration feedforward:

$$
\boldsymbol{a}^{\boldsymbol{b}}=\ddot{\boldsymbol{\eta}}_{\boldsymbol{d}}-\boldsymbol{K}_{\boldsymbol{d}} \dot{\tilde{\boldsymbol{\eta}}}-\boldsymbol{K}_{\boldsymbol{P}} \tilde{\boldsymbol{\eta}}-\boldsymbol{K}_{\boldsymbol{i}} \int_{0}^{t} \tilde{\boldsymbol{\eta}}(\tau) d \tau
$$

where $\boldsymbol{K}_{\boldsymbol{p}}, \boldsymbol{K}_{\boldsymbol{d}}$ and $\boldsymbol{K}_{\boldsymbol{i}}$ are positive definite gain matrices and $\tilde{\boldsymbol{\eta}}$ is the position error vector. For simplification 5 assumes stable pitch and roll angles. The PID is implemented with integral windup limit. The control input $\tau$ has a saturation limit and rate limit to match the actuation capabilities of the robot. We tuned the gains for depth and yaw in still water using trial and error method.

The values of the matrices $C(\nu), D(\nu)$ and $g(\eta)$ are calculated in a separate independent node depending on the measured position and velocity of the vehicle. This separation allows very rapid implementation of different model-based controllers.

\section{B. Human intervention}

In addition to the automatic control, all DOFs can be controlled manually using a joystick. Joystick outputs axis values between 0 to 1 . These values are proportionally mapped to manual control input $\tau_{m}$ (see Joystick node on Fig. 2. For each DOF, operator can choose which control input the vehicle uses, manual or automatic.

\section{Priority management}

To prioritize the DOFs, control input vector $\tau$ elements are multiplied by priority vector $\boldsymbol{p}=\left[p_{x}, p_{y}, p_{z}, p_{\varphi}, p_{\vartheta}, p_{\psi}\right]$ elements, resulting in prioritized control input vector $\tau_{p}$. The priority of each DOF varies from 0 to 1 . To assign priorities, different sets of laws could be created. Our tests show that on U-CAT it is best to use laws that assign priorities depending on the control action of a single DOF. We created laws that give highest priority to yaw and depth control when surge action is small. When Surge action increases, the priority of depth and yaw decreases. In principle, the control is modified depending on what motion the vehicle is about to perform. When the vehicle is close to its desired $x, y$ position, it can precisely hover and orient itself towards desired direction. When a new waypoint is published or when the operator wants to move forward, surge action increases and the action of depth and yaw decreases. The laws we use are the following:

$$
\begin{gathered}
p_{z}=p_{\psi}=c_{1}\left|\alpha_{x}\right|^{3}+c_{2}\left|\alpha_{x}\right|^{2}+c_{3}\left|\alpha_{x}\right|+c_{4} \\
p_{x}=1-p_{z}
\end{gathered}
$$

where $\alpha_{x}=\tau_{x} / \tau_{x \max }$ is the normalized control input. Constants $c_{i}$ are such that $0<\alpha_{1}<1 \rightarrow 0<p_{z}<1$. The rules are plotted in Fig. 2.

\section{Wrench driver}

The wrench driver converts the generalized, priortized control input $\boldsymbol{\tau}_{\boldsymbol{p}}$ into fin actuation parameters $A_{i}, f_{i}$ and $\Phi_{i}$. It first uses a set or rules to convert forces and moments of different DOFs to corresponding fin thrust forces $F_{i}$. We have defined different rule sets for different types of locomotion (i.e. fast swimming or precise hovering). In this study we use only one fixed set of rules, which we call the precise hovering mode. Similar approach is also implemented in [10]. In the precise hovering mode, the fore and aft fins are always pointing towards each-other. When the robot needs a forward surge motion, only fore fins are actuated. In case of reverse surge motion only aft fins are actuated. For clockwise yaw fore left and aft right fins are actuated and so on. In this configuration the U-CAT can continuously create thrust forces in all 6 DOFs without a need to reorient its fins 180 degrees. The conversion from fin thrust forces to actuation parameters is found from (2). We use a fixed frequency of 2 $\mathrm{Hz}$ and only vary the oscillation amplitudes $A_{i}$ and directions $\Phi_{i}$. Wrench driver forwards the actuation parameters to Maxon motor controllers who handle the position control of fins.

\section{E. Odometry and trajectory}

Odometry node calculates the positions and velocities of the vehicle using the feedback from IMU and pressure sensor. It also estimates the position of the vehicle in the worldfixed frame using the dynamics of the vehicle. However, we do not use this estimation in the current study due to a lack of a good state observer.

The trajectory manager creates smooth position and velocity trajectories. In the current study it uses predefined timed setpoints written in a file. It then uses hyperbolic tangent functions to calculate smooth transitions between setpoints. The trajectory manager is also able to read setpoints from other sources. For example remote controller can be used to modify the desired depth and yaw.

\section{EXPERIMENTS IN POOL}

To validate our control approach we conducted experiments with different controller configurations. We did the experiments in a $60 \mathrm{~m}$ long, $5 \mathrm{~m}$ wide and $3 \mathrm{~m}$ deep fresh water tow tank of Tallinn University of Technology. In all the experiments the robot had to follow a lawnmower trajectory at constant depth of $1 \mathrm{~m}$. Each experiment started with a dive and ended with a resurface. We chose the lawnmower trajectory because of its wide use in underwater inspection tasks. During the experiments the robot recorded its depth and yaw data into ROS log files. As the acoustic positioning methods did not work in a concrete tank, we didn't have any real-time position feedback for translational motions on horizontal plane. However, we used a calibrated overhead camera for recording the vehicle trajectories. After 
the experiments we manually tracked the position of U-CAT from the video frame by frame.

We tested 5 different control configurations to show the advantages of our control approach: manual control; manual control with depth autopilot; manual control with depth and yaw autopilot; fully autonomous control; fully autonomous control without DOF prioritization. The desired and achieved trajectories for each experiment are shown in Fig. 3. Root mean square (RMS) error values calculated over the full length of experiments are marked on graphs.

\section{A. Experiment 1: Manual control}

For comparison, we first tested the trajectory following using manual control. An experienced U-CAT operator controlled depth, yaw and surge to follow the predefined trajectory as precisely as possible while keeping constant depth. The operator used visual feedback from the poolside and a depth reading on screen. The lawnmower trajectory was relatively loosely defined in our experiments, as there was no precise position feedback other than visual. The operator used markers on the poolside to approximately travel a same distance with every short leg of the trajectory. For comparative reasons the operator also tried to follow the same trajectory timing as in other experiments.

Fig. 3a shows the desired and actual trajectories of depth, yaw, $x$ and $y$. From the figure it can be easily seen that the trajectory following precision is poor (depth RMS error of $27.6 \mathrm{~cm}$ and yaw RMS error of $39.8^{\circ}$ ). This illustrates the definite need for at least some autopiloting.

\section{B. Experiment 2: Depth autopilot}

In the second experiment the depth autopilot was enabled. Operator manually controlled the surge and yaw. Results show $81 \%$ decrease in depth error. In addition yaw error decreased $28 \%$ as now the operator had to concentrate on controlling only 2 DOFs instead of 3 . However, the overhead camera image still reveals the lack of precision in tracking straight lines. The vehicle turns rapidly. This kind of unsteady motion would probably cause problems in video inspection tasks, especially when using automatic video processing methods such as mosaicking.

\section{Experiment 3: Depth and yaw autopilot}

In this experiment we also enabled automatic yaw control. This time the operator intervention was minimal. He only had to control the surge motion to move the vehicle from one turning point to another. Results (Fig. 3c) show high precision in yaw tracking (RMS error of $2.5^{\circ}$; improvement of $91 \%$ ). Also, due to more steady yaw the depth tracking error decreased by $63 \%$. More stable yaw is also visible from the overhead camera image. It shows how the robot moves in straight lines between turning points. Unfortunately, the short legs of the trajectory (vertical on the overhead camera images) are not perpendicular to the long legs. This is caused by the disturbance of the tether, which slowly pulled the vehicle sideways. The disturbances are inevitable and during ROV missions they have to be manually compensated for. The compensation can be relatively easily done as the U-CAT's fins also allow to actuate the sway motion to move the vehicle sideways. In this article, however, we do not consider sway control.

\section{Experiment 4: Fully autonomous control}

As U-CAT is designed to eventually be fully autonomous, we also tested the extendibility of our control approach to fully autonomous mode. In this case the vehicle was untethered and the operator was removed from the control loop. The manual surge regulation was replaced by automatic open-loop control. As we didn't have any surge velocity or position feedback, the surge control was implemented as a smooth predefined force trajectory. The results (Fig. 3d) show that removing human intervention did not significantly reduce the tracking precision. Also, the tether disturbance is not any more visible on the overhead image. However, the figure reveals an IMU drift in yaw signal which was not present in our previous experiments. We have noticed that large IMU drift sometimes occurs due to electromagnetic noise emitted by the motor controllers. The drift is small enough $(0.9 \mathrm{deg} / \mathrm{min})$ to not affect the controller behaviour. For real missions the drift will be reduced by minimizing electromagnetic noise and by using magnetometers and Extended Kalman Filter.

Despite the IMU issue the experiment shows that our control approach is suitable for simultaneously controlling 3 DOFs of the fully autonomous 4-fin vehicle. Once the vehicle has a reliable position or surge velocity estimation, the current surge controller can be replaced with a suitable closed-loop controller.

\section{E. Experiment 5: Fully autonomous control without DOF prioritization}

We conducted another experiment with a goal to justify the usage of DOF priority functions. In this experiment we repeated the fully autonomous control scenario, but we disabled the priority functions. The results (Fig. 3e) show strong oscillations of depth value. The oscillations occur when the surge action increases and the vehicle tries to move forward. The figure also displays the roll and pitch angles in comparison to those during the previous experiment (Fig. $3 \mathrm{~d})$. It can be seen that the vehicle is strongly oscillating in pitch and roll. The oscillations are a result of a coupling between the actuation of different DOFs. All the controllers try to stabilize the vehicle, however the fin configuration does not allow to equally control all the DOFs at the same time. The resulting lawnmower trajectory is also different from that of the experiment 4 as the vehicle can not correctly output the predefined surge force. These results clearly show the importance of DOF prioritization in our system.

\section{CONCLUSION}

Our study reveals several advantages of the proposed control architecture. Comparative experiments show that the problems arising from coupled DOFs can be avoided using priority functions. Priority functions allowed us to develop 

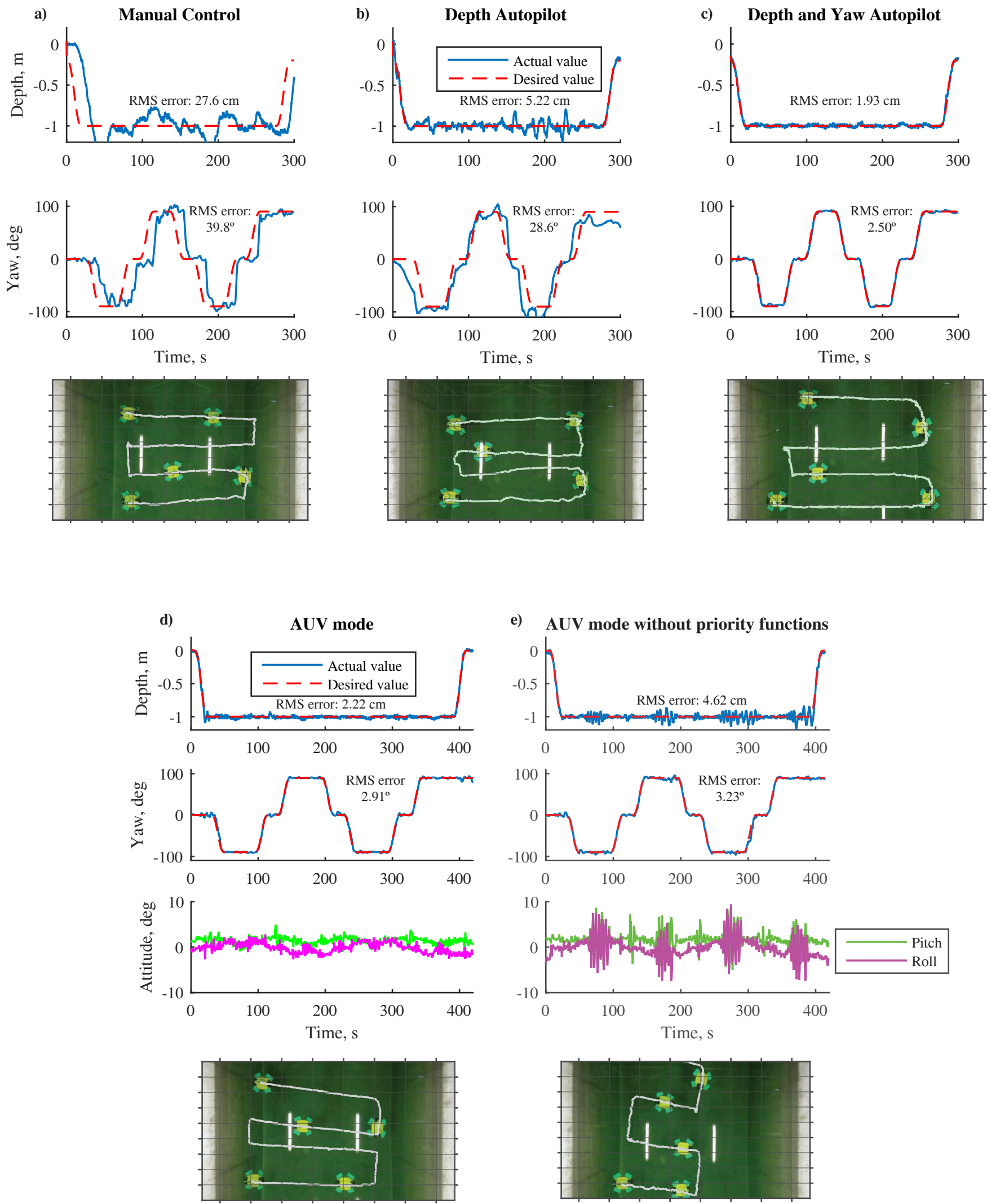

Fig. 3. Experimental results 
a modular control system, which in turn helps to reduce the complexity of the control. Control parameter tuning of U-CAT was a straightforward and simple process, but it still resulted in high trajectory tracking precision. We showed that the system can be easily applied for different control scenarios, such as ROV autopiloting or 3DOF AUV mode. The modularity of the approach allows to easily extend it for different scenarios. The controllers for different DOFs can be separately replaced with more suitable ones if needed, for example adaptive control could be used for depth if the robot should be equipped with a manipulator. Our approach is limited to cases when steady and stable vehicle motion is required. It is probably not easily extensible to situations where more complex and agile movements are desired.

As a next step we plan to increase the number of automatically controlled DOFs. We are currently working on a development a proper absolute $x, y$ position feedback. For this we will use different localization sensors of the U-CAT, state observer and extended Kalman filters. Position feedback allows us to add automatic control for surge and sway motion. In addition we are working on the implementation and testing of different controllers to find the best approach for different scenarios. For example we intend to use adaptive tuning of the model parameters during operation. Adaptive parameters would compensate for the uncertainties, such as the buoyancy of the tether.

\section{ACKNOWLEDGMENT}

This research has received funding from the European Union's Seventh Framework Programme for Research technological development and demonstration, under grant agreement no. 308724 (The ARROWS Project), from EstonianFrench joint collaboration project PHC-PARROT and from IUT339 grant of Estonian Ministry of Education and Research. The authors would like to thank Keijo Kuusmik, Jaan Rebane, Riho Markna who have helped to develop the UCAT vehicle. We would also like to thank TUT Small Craft Competence Centre for letting us use the tow tank.

\section{REFERENCES}

[1] Y.-S. Ryuh, G.-H. Yang, J. Liu, and H. Hu, "A school of robotic fish for mariculture monitoring in the sea coast," Journal of Bionic Engineering, vol. 12, no. 1, pp. 37-46, 2015.

[2] A. Kohl, K. Pettersen, E. Kelasidi, and J. Gravdahl, "Planar path following of underwater snake robots in the presence of ocean currents," Robotics and Automation Letters, IEEE, vol. PP, no. 99, pp. 1-1, 2016.

[3] R. J. Lock, R. Vaidyanathan, S. C. Burgess, and J. Loveless, "Development of a biologically inspired multi-modal wing model for aerialaquatic robotic vehicles through empirical and numerical modelling of the common guillemot, uria aalge," Bioinspiration \& biomimetics, vol. 5, no. 4, p. 046001, 2010.

[4] A. Konno, T. Furuya, A. Mizuno, K. Hishinuma, K. Hirata, and M. Kawada, "Development of turtle-like submergence vehicle," in Proceedings of the 7th International Symposium on Marine Engineering, 2005.

[5] K. Low, C. Zhou, T. Ong, and J. Yu, "Modular design and initial gait study of an amphibian robotic turtle," in Robotics and Biomimetics, 2007. ROBIO 2007. IEEE International Conference on. IEEE, 2007, pp. 535-540.

[6] S. C. Licht, "Biomimetic oscillating foil propulsion to enhance underwater vehicle agility and maneuverability," Ph.D. dissertation, Massachusetts Institute of Technology, Cambridge, Massachusetts, June 2008.
[7] C. Siegenthaler, C. Pradalier, F. Gunther, G. Hitz, and R. Siegwart, "System integration and fin trajectory design for a robotic seaturtle," in Intelligent Robots and Systems (IROS), 2013 IEEE/RSJ International Conference on. IEEE, 2013, pp. 3790-3795.

[8] J. D. Geder, R. Ramamurti, M. Pruessner, and J. Palmisano, "Maneuvering performance of a four-fin bio-inspired uuv," in Oceans-San Diego, 2013. IEEE, 2013, pp. 1-7.

[9] J. D. Geder, R. Ramamurti, D. Edwards, T. Young, and M. Pruessner, "Development of a robotic fin for hydrodynamic propulsion and aerodynamic control," in Oceans-St. John's, 2014. IEEE, 2014, pp. $1-7$.

[10] M. Kemp, B. Hobson, and J. H. Long, "Madeleine: an agile auv propelled by flexible fins," in Proceedings of the 14th International Symposium on Unmanned Untethered Submersible Technology, vol. 6, 2005.

[11] J. H. Long Jr, J. Schumacher, N. Livingston, and M. Kemp, "Four flippers or two? tetrapodal swimming with an aquatic robot," Bioinspiration \& Biomimetics, vol. 1, no. 1, p. 20, 2006.

[12] S. Licht and N. Durham, "Biomimetic robots for environmental monitoring in the surf zone \& in very shallow water," in IEEE/RSJ International Conference on Intelligent Robots and Systems, 2012.

[13] G. Yao, J. Liang, T. Wang, X. Yang, Q. Shen, Y. Zhang, H. Wu, and W. Tian, "Development of a turtle-like underwater vehicle using central pattern generator," in Robotics and Biomimetics (ROBIO), 2013 IEEE International Conference on. IEEE, 2013, pp. 44-49.

[14] C. Wang, G. Xie, X. Yin, L. Li, and L. Wang, "Cpg-based locomotion control of a quadruped amphibious robot," in Advanced Intelligent Mechatronics (AIM), 2012 IEEE/ASME International Conference on. IEEE, 2012.

[15] W. Zhao, Y. Hu, L. Wang, and Y. Jia, "Development of a flipper propelled turtle-like underwater robot and its cpg-based control algorithm," in Decision and Control, 2008. CDC 2008. 47th IEEE Conference on. IEEE, 2008, pp. 5226-5231.

[16] G. Dudek, P. Giguere, J. Zacher, E. Milios, H. Liu, P. Zhang, M. Buehler, C. Georgiades, C. Prahacs, S. Saunderson et al., "Aqua: An amphibious autonomous robot," Computer, no. 1, pp. 46-53, 2007.

[17] N. Plamondon and M. Nahon, "Adaptive controller for a biomimetic underwater vehicle," Journal of Unmanned Vehicle Systems, vol. 1, no. 01 , pp. 1-13, 2013.

[18] — - "A trajectory tracking controller for an underwater hexapod vehicle," Bioinspiration \& biomimetics, vol. 4, no. 3, p. 036005, 2009.

[19] N. Plamondon, "Modeling and control of a biomimetic underwater vehicle," Ph.D. dissertation, McGill University, Montreal,Quebec, January 2010.

[20] P. Giguere, Y. Girdhar, and G. Dudek, "Wide-speed autopilot system for a swimming hexapod robot," in Computer and Robot Vision (CRV), 2013 International Conference on. IEEE, 2013, pp. 9-15.

[21] D. Meger, F. Shkurti, D. Cortes Poza, P. Giguere, and G. Dudek, "3d trajectory synthesis and control for a legged swimming robot," in Intelligent Robots and Systems (IROS 2014), 2014 IEEE/RSJ International Conference on. IEEE, 2014, pp. 2257-2264.

[22] Y. Girdhar and G. Dudek, "Exploring underwater environments with curiosity," in Computer and Robot Vision (CRV), 2014 Canadian Conference on. IEEE, 2014, pp. 104-110.

[23] (2016) Fp7 arrows project website. [Online]. Available: http://www.arrowsproject.eu/

[24] T. Salumäe, R. Raag, J. Rebane, A. Ernits, G. Toming, M. Ratas, and M. Kruusmaa, "Design principle of a biomimetic underwater robot u-cat," in Oceans-St. John's, 2014. IEEE, 2014, pp. 1-5.

[25] A. Chemori, K. Kuusmik, T. Salumäe, and M. Kruusmaa, "Depth control of the biomimetic u-cat turtle-like auv with experiments in real operating conditions," in Robotics and Automation (ICRA), 2016 IEEE International Conference on. Stockholm, Sweden: IEEE, May 2016, pp. 1-5.

[26] T. I. Fossen, Handbook of marine craft hydrodynamics and motion control. John Wiley \& Sons, 2011.

[27] B. Siciliano and O. Khatib, Springer handbook of robotics. Springer Science \& Business Media, 2008.

[28] A. R. Chowdhury, V. Vishwanathan, B. Prasad, R. Kumar, and S. K. Panda, "Inverse dynamics control of a bio-inspired robotic-fish underwater vehicle propulsion based on lighthill slender body theory," in OCEANS 2014 - TAIPEI, April 2014, pp. 1-6. 